\title{
1 Ecogenomics of groundwater viruses suggests niche differentiation linked to specific
}

\section{2 environmental tolerance}

3 Ankita Kothari ${ }^{\mathrm{a}}$, Simon Roux ${ }^{\mathrm{b}}$, Hanqiao Zhang ${ }^{\mathrm{a}}$, Anatori Prieto ${ }^{\mathrm{a}}$, Drishti Soneja ${ }^{\mathrm{a}}$, John-Marc

4 Chandonia $^{\mathrm{a}}$, Sarah Spencer ${ }^{\mathrm{h}, \mathrm{i}, \mathrm{k}}$, Xiaoqin $\mathrm{Wu}^{\mathrm{d}}$, Sara Altenburg ${ }^{1}$, Matthew W. Fields ${ }^{1, \mathrm{~m}}$, Adam M.

5 Deutschbauer ${ }^{\mathrm{c}, \mathrm{e}}$, Adam P. Arkin ${ }^{\mathrm{c}, \mathrm{f,g}}$, Eric J. Alm ${ }^{\mathrm{h}, \mathrm{i}, \mathrm{j}, \mathrm{k}}$, Romy Chakraborty ${ }^{\mathrm{d}}$, Aindrila

6 Mukhopadhyay, ${ }^{\mathrm{a}, \#}$

8 abiological Systems and Engineering, Lawrence Berkeley National Laboratory, Berkeley, 9 California, USA.

10 bDepartment of Energy Joint Genome Institute, Lawrence Berkeley National Laboratory, 11 Berkeley, California, USA.

$12{ }^{c}$ Environmental Genomics and Systems Biology Division, Lawrence Berkeley National 13 Laboratory, Berkeley, California, USA.

14 dEarth and Environmental Sciences, Lawrence Berkeley National Laboratory, Berkeley, 15 California, USA

16 e Department of Plant and Microbial Biology, University of California, Berkeley, California,

17 USA

18 fEnergy Biosciences Institute, Berkeley, California, USA

19 gDepartment of Bioengineering, University of California, Berkeley, California, USA

20 hepartment of Civil and Environmental Engineering, Massachusetts Institute of Technology,

21 Cambridge, MA, USA

22 iDepartment of Biological Engineering, Massachusetts Institute of Technology, Cambridge,

23 Massachusetts, USA

24 jBroad Institute of MIT Cambridge, Cambridge, Massachusetts, USA

$25{ }^{\mathrm{k}}$ Center for Microbiome Informatics and Therapeutics, Massachusetts Institute of Technology, 26 Cambridge, Massachusetts, USA

27 'Sara Altenburg, Center for Biofilm Engineering, Montana State University, Montana, USA

28 mepartment of Microbiology \& Immunology, Montana State University, Montana, USA 
32 Running title: Ecogenomics of groundwater viruses

33

34 \#Address correspondence to Aindrila Mukhopadhyay, amukhopadhyay@lbl.gov.

36 Keywords: groundwater, virus, phage, plasmidome, viral sequences, metal resistance, antibiotic resistance.

\section{Abstract}

Viruses are ubiquitous microbiome components, shaping ecosystems via strain-specific predation, horizontal gene transfer and redistribution of nutrients through host lysis. Viral impacts are important in groundwater ecosystems, where microbes drive many nutrient fluxes and metabolic processes, however little is known about the diversity of viruses in these environments. We analyzed four groundwater plasmidomes and identified 200 viral sequences, which clustered into $41 \sim$ genus-level viral clusters (equivalent to viral genera) including 9 known and 32 putative new genera. We use publicly available bacterial whole genome sequences (WGS) and WGS from 261 bacterial isolates from this groundwater environment to identify potential viral hosts. We linked 76 of the 200 viral sequences to a range of bacterial phyla, the majority associated with Proteobacteria, followed by Firmicutes, Bacteroidetes and Actinobacteria. The publicly available microbial genome sequences enabled mapping bacterial hosts to a breadth of viral sequences. The WGS of groundwater isolates increased depth of host prediction by allowing identification of hosts at the strain level. The latter included 4 viruses that were almost entirely ( $>99 \%$ query coverage, $>99 \%$ identity) identified as integrated in the genomes of specific Pseudomonas, Acidovorax and Castellaniella strains, resulting in very high-confidence host assignments. Lastly, 21 of these viruses encoded putative auxiliary metabolite genes for metal and antibiotic resistance, which might drive their infection cycles and/or provide selective advantage to infected hosts. Exploring the groundwater virome provides a necessary foundation for integration of viruses into ecosystem models where they act as key players in microbial adaption to environmental stress.

\section{Importance}

61 To our knowledge, this is the first study to identify the bacteriophage distribution in a groundwater 
63 background sites. Our study is uniquely based on selective sequencing of solely the 64 extrachromosomal elements of a microbiome followed by analysis for viral signatures, thus establishing a more focused approach for phage identifications. Using this method, we detect several novel phage genera along with those previously established. Our approach of using the whole genome sequences of hundreds of bacterial isolates from the same site enabled us to make host assignments with high confidence, several at strain levels. Certain phage-encoded genes suggest they provide an environment-specific selective advantage to their bacterial hosts. Our study lays the foundation for future research on directed phage isolations using specific bacterial host strains to further characterize groundwater phages, their lifecycles, and its effects on groundwater microbiome and biogeochemistry.

\section{Introduction}

Viruses are known to influence the structure and diversity of microbial communities by infection and lysis of microbial cells. Their influence has been widely studied in aquatic communities ${ }^{1}$ where they are predicted to infect approximately one-third of seawater microbes at any given time ${ }^{2}$. In marine ecosystems, major biogeochemical cycles are known to be influenced by viruses affecting community composition, metabolic activity, and evolutionary trajectories ${ }^{2,3}$. As the recent focus on exploration of viruses in aquatic environments has been on marine ecosystems ${ }^{4-9}$, fresh water environments remained mostly unexplored despite their importance as drinking water supply ${ }^{10}$. The Oak Ridge Field Research Center (ORFRC) ${ }^{11-13}$ is a well-studied United States Department of Energy site that includes groundwater areas with and without metal contamination, referred to as the contaminated and background sites, respectively. It has been well characterized in terms of the physical parameters, microbiome distribution and fluctuation in response to different environmental stresses and thus served as an excellent model groundwater system for studies. We chose this environment to study the incidence of viruses in groundwater microbiome.

Identification of viral sequences in the environment is difficult given the lack of approaches similar to ribosomal DNA profiling in bacteria and their isolation remains challenging because of the difficulties in identifying the bacterial host(s) and our limited ability to culture them. Recently, 
94 circumventing these limitations and providing direct insights into the composition of 95 environmental viral communities ${ }^{16}$. In this study we explore an alternate method to sifting through large amounts of chromosomal DNA sequences to find viral sequences by specifically searching circular DNA sequence data generated from the plasmidome analysis. Specifically, we mined the plasmidome data from a well-characterized groundwater system and analyzed the resulting viral sequences complete with genomic and ecological contexts.

\section{Methods}

\subsection{Groundwater sample collection and sequencing analysis}

The groundwater samples were obtained from Oak Ridge Field Research Center (ORFRC) site ${ }^{11-}$ 13 and included metal-contaminated (wells FW104 and FW106) and background (wells GW456 and GW460) areas. An earlier study ${ }^{17}$ described the circular DNA isolation (plasmidome analysis) procedure from 4 liters of groundwater from background sites (GW456 and GW460), followed by sequencing, assembly, annotation and other analyses. Additionally, for this present study, we also use plasmidome sequence data from two contaminated site samples comprised of 8 liters ground water from FW104 and FW106 and subjected to the same analysis (manuscript in preparation, sequencing data available via MG-RAST IDs mgm4830571.3 and mgm4830867.3).

111

\subsection{Identification of viral contigs}

113 Post sequencing, the assembly of all contigs (including plasmid and viral DNA), along with 114 prediction of circular sequences using bioinformatic analyses were performed as described 115 previously $^{17}$. Briefly, all plasmid sequences obtained were subjected to a pipeline method for

116 postassembly detection of circularity among scaffolds, and any scaffolds failing this are termed as

117 non-circular contigs, to distinguish them from those plasmid sequences which passed the criteria.

118 All circular contigs along with non-circular contigs encoding more than 10 proteins were subjected

119 to VirSorter analysis ${ }^{18}$, an iVirus tool available via Cyverse ${ }^{19}$ for identification of viruses. 120 VirSorter was used to identify and remove microbial contigs using the 'virome decontamination'

121 mode, with every contig that was not identified as viral considered to be a microbial contig. The 122 final set of viral contigs was formed by compiling sequences detected as VirSorter categories 1 123 and 2 along with prophage categories 4 and 5 (Table S1). Thus, we focus on the 200 viral sequences 124 with high confidence assignments (VirSorter categories 1,2 4, and 5), and ignored the low 
125 confidence assignments (VirSorter categories 3 and 6). Vcontact $2^{20}$ was used to perform viral

126 cluster analysis, and the results were visualized using cytoscape ${ }^{21}$. Since the groundwater from

127 background site was spiked with strains Desulfovibrio vulgaris Hildenborough (ATCC 29579),

128 Escherichia coli DH1 (ATCC 33849), and E. coli strain J-2561 as controls for the plasmidome

129 study $^{17}$, any viruses associated with these strains were removed from the analysis. Given that the

130 DNA isolation procedure concentrated on targeted isolation of circular DNA, there is an expected

131 inherent bias in identifying circular dsDNA viral sequences from this dataset.

132

$133 \quad 2.2$ Generation of host database

\section{$134 \quad$ 2.2.1 Generation of host database from ORFRC bacterial isolates}

135 Isolation of bacterial strains

136 The bacterial isolates were obtained via direct-plating under aerobic or anaerobic conditions at 25-

$13730{ }^{\circ} \mathrm{C}$ in the dark, using ORFRC groundwater or sediment extract as inoculum, or via two-step

138 isolation: enrichment incubation of $1 \mathrm{ml}$ groundwater in $9 \mathrm{ml}$ liquid media aerobically for two

139 weeks followed by direct-plating for isolation. A subset of isolates were obtained from biofilm

140 reactors (CDC reactors) that were fed ORFRC groundwater and had non-porous glass beads (30

$141 \mathrm{um}$ ) as matrix for biofilms in coupons. Water or beads from the reactors were used as inoculum.

142 For direct-plating, rich media (Luria-Bertani, tryptic soy, R2A, Eugon, Winogradsky) agar plates,

143 or basal medium (4.67 $\mathrm{mM}$ ammonium chloride, $30 \mathrm{mM}$ sodium phosphate, with vitamin and

144 mineral mixes as previously described ${ }^{22}$ ) agar plates were used. The liquid media for enrichment

145 incubation was filtered groundwater amended with one or a combination of the following carbon

146 sources: glucose $(5 \mathrm{mM})$, acetate $(5 \mathrm{mM})$, benzoate $(0.5 \mathrm{mM})$, casamino acid $(10 \mu \mathrm{g} / \mathrm{ml})$, bacterial

147 cell lysate, and sediment-extracted dissolved organic matter. After direct-plating, single colonies

148 were picked and regrown in liquid media for 16-48 $\mathrm{h}$ until the culture reached mid-log phase. Then

149 a portion of the culture was used to extract DNA for 16S rRNA based identification, and the rest

150 were cryopreserved with sterile glycerol (to a final concentration of 30\%), flash frozen with liquid

151 nitrogen, and stored at $-80^{\circ} \mathrm{C}$.

152

153 Whole genome sequencing and de novo assembly

154 Cultures were revived from glycerol stocks by streaking onto Luria-Bertani or R2A agar plates.

155 Individual colonies developed at $30^{\circ} \mathrm{C}$ over $48 \mathrm{~h}$, which were then inoculated into corresponding 
156 liquid media and grown at $30^{\circ} \mathrm{C}$ for 48 hours. The cultures were centrifuged, the genomic DNA 157 was extracted using the Qiagen DNeasy kit (Qiagen, Venlo, NL) according to the manufacturer's 158 instructions. All samples were eluted in Qiagen's AE buffer: $10 \mathrm{mM}$ Tris-Cl, $0.5 \mathrm{mM}$ EDTA, pH 159 9.0. Genomic DNA was stored at $-20^{\circ} \mathrm{C}$ followed by transfer into a 384-well plate for automated 160 library preparation. The isolated genomic DNA was normalized to $0.2 \mathrm{ng} / \mathrm{uL}$ in $10 \mathrm{mM}$ Tris (pH 161 8.0), and libraries were prepared using the Illumina Nextera XT kit at 1/12th reaction size on a 162 SPT Labtech Mosquito HV. Final libraries were purified using Solid Phase Reversible 163 Immobilization beads, and sequenced on an Illumina NextSeq 500 with 150 bp paired-end reads.

164 The program Cutadapt v1.12 was used to remove adapter sequences with parameters -a 165 CTGTCTCTTAT -A CTGTCTCTTAT ${ }^{23}$. We performed sliding window quality filtering with 166 Trimmomatic v0.36 (parameters -phred33 LEADING:3 TRAILING:3 SLIDINGWINDOW:5:20 MINLEN:50) ${ }^{24}$. All genomes were assembled de novo using SPAdes v3.9.0 with the following options (-k 21,33,55,77 --careful) ${ }^{25}$. Genome quality was validated with the program checkM v1.0.6 using the lineage_wf pipeline with default parameters ${ }^{22}$, and all draft genomes passed the criteria of contamination $<10 \%$ and completeness $>95 \%$. The $16 \mathrm{~S}$ rRNA gene sequences were recovered with RNAmmer v1.2 ( $-\mathrm{S}$ bac $-\mathrm{m}$ ssu) and taxonomically classified with SINTAX

172 (usearch v9.2.64) against the Ribosomal Database Project (RDP) ${ }^{28}$ 16S rRNA gene training set 173 v16 with species names and the following parameters (-strand both - sintax_cutoff 0.8$)^{26,27}$. The

174 whole genome sequences (WGS) of 261 bacterial isolates (details in Table S2) from ORFRC were combined to form a database for further bioinformatic analyses. The WGS of the 261 strains are available via (https://kbase.us/n/63776/35) and the DOI (10.25982/63776.53/1637360).

\subsubsection{Generation of host database from NCBI bacterial and archaeal isolates}

A genome database of putative hosts for the viruses was generated including all archaeal (311 assembled complete genomes, downloaded in September 2019) and bacterial (14028 assembled complete genomes, downloaded in August 2019) genomes from NCBI Assembly. The taxonomic affiliation of the genomes was taken from the NCBI taxonomy.

\subsection{Host prediction and diversity}

185 Three different previously published approaches ${ }^{29,30}$ for predicting hosts based on examining 
187 microbial genomes due to integrated prophages or gene transfers ${ }^{32}$ and c) viral and host genome 188 nucleotide signatures (here, tetranucleotide frequency similarity) ${ }^{33}$ were used as described below.

189 The confidence in assignment via these three methods to different clades in bacterial classification

190 has been previously estimated ${ }^{34}$ with CRISPR-based predictions being the most accurate while the

191 tetranucleotide frequency-based predictions were the least accurate at the genus level.

192

\section{BLAST-based identification of sequence similarity between viral contigs and host genome}

All 200 viral contigs were compared to all archaeal and bacterial genomes with BLASTn (threshold of 50 for bit score and 0.001 for $E$-value), to identify regions of similarity between a viral contig and a microbial genome, indicative of a prophage integration or horizontal gene transfer. As previously established ${ }^{30}$, host prediction was made when an NCBI genome displayed a region similar to the viral contig $\geq 4.9 \mathrm{~kb}$ at $\geq 70 \%$ identity. When one viral sequence had hits to multiple bacterial strains, the top 5 hits (based on bit score) were analyzed to determine the last common ancestor clade. This clade was then assigned as the host to the virus. Based on this methodology, genus level bacterial host predictions were made. Bacterial strain-specific host predictions were only made when the entire virus was found to be encoded in the bacterial whole genome sequence. In this case, BLAST with highly stringent parameters, referred to as BLAST99 ( $>99 \%$ query coverage, e-value $=0$ and $>99 \%$ identity) was performed to query for the presence of an entire viral sequence in the host.

\section{Matches between viral contigs and CRISPR spacers}

CRISPR arrays were predicted for all ORFRC microbial genomes with CRISPR Recognition Tool, 38 maximum repeat length, and a search window of 8 ; along with spacer settings used 19 minimum

211 spacer length and 48 maximum spacer length). We used previously published ${ }^{30,} 36$ BLAST 212 parameters for identifying the target of CRISPR spacers (i.e. using the BLASTn-short task, a 213 maximum expect value of 1; a gap opening penalty 10; a gap extension penalty 2; a word size 7; 214 and dust filtering turned off). Given that the accuracy of this approach for detecting phage hosts 215 strongly depends on the maximum number of mismatches allowed between the CRISPR spacer 216 and the viral sequence, the results were filtered to allow 0 or 1 mismatch. Only the CRISPR spacers

217 that matched viral sequences were then compared back with the bacterial WGS with no mismatch 
218 to come up with bacterial host predictions. Based on this methodology, strain level bacterial host

219 predictions were made.

220

\section{Nucleotide composition similarity: comparison of tetranucleotide frequency}

222 Bacterial and archaeal viruses tend to have a genome composition close to the genome composition 223 of their host, a signal that can be used to predict viral-host pairs ${ }^{30,33,37}$. Here, canonical 224 tetranucleotide frequencies (also referred to as 4mer) were observed for all viral and host sequences 225 using Jellyfish ${ }^{38}$ and mean absolute error (that is, the average of absolute differences) between 226 tetranucleotide-frequency vectors were computed with in-house Perl and Python scripts for each 227 pair of viral and host sequence as previously reported ${ }^{34}$. A viral contig was then assigned if the average of absolute differences $(d)$ between tetranucleotide-frequency vectors $d<0.001$. When multiple strains had hits to one viral sequence, the top five hits (based on lowest distance) were analyzed to determine the lowest common ancestor to the group. This lowest common ancestor was then assigned as the host to the virus. Based on this methodology, genus level bacterial host predictions were made.

233

\subsection{Phylogenetic tree construction}

235 For constructing the phylogenetic tree using ORFRC isolates, the 16S rRNA sequences from all 236261 strains were aligned using Muscle $^{39}$. The evolutionary history was inferred by using the 237 Maximum Likelihood method based on the Tamura-Nei model using MEGA $7^{40}$. The tree with the 238 highest log likelihood (-7846.44) is shown. Initial tree(s) for the heuristic search were obtained 239 automatically by applying Neighbor-Join and BioNJ algorithms to a matrix of pairwise distances 240 estimated using the Maximum Composite Likelihood (MCL) approach, and then selecting the 241 topology with superior log likelihood value. The analysis involved 255 nucleotide sequences. All 242 positions containing gaps and missing data were eliminated. There were a total of 519 positions in 243 the final dataset. All branches were collapsed at the genus level. For the phylogenetic tree depicting 244 NCBI isolates, existing trees were downloaded using NCBI taxonomy and collapsed to genus 245 levels. 
A functional annotation of all virus-encoded predicted proteins was based on a comparison to the Pfam domain database v. $32^{41}$ with $\mathrm{HmmScan}^{42}$ (threshold of 30 for bit score and $10^{-3}$ for $E$-value).

250 The Pfam categories were assigned based on Pfam target name as previously described ${ }^{34}$, and any 251 Pfam target name not categorized earlier is referred to as "not categorized". All contigs were also 252 uploaded to KBase for annotation. To specifically identify metal and antibiotic resistance genes, 253 all the unique Pfam target names and their descriptions were manually curated.

\section{Results and Discussion}

\section{New viruses detected in the circular DNA datasets}

To study groundwater viruses, we leveraged existing data focused on extrachromosomal circular DNA templates by identifying viruses from plasmidome datasets (Fig 1). Viruses and plasmids can coexist stably, support the transfer of each other to new hosts ${ }^{43}$ or even form a hybrid ${ }^{44}$. Given that both can be found as extrachromosomal circular DNA molecules, we used VirSorter, a tool designed to predict bacterial and archaeal virus sequences on the plasmidome assemblies ${ }^{18}$ and identified 200 sequences as groundwater viral sequences from 13,770 plasmidome contigs (Fig $\mathrm{S} 1$ ). We then categorized viral sequences into viral clusters (approximately equivalent to known viral genera) using shared gene-content information and network analytics ${ }^{33}, 45$. Clustering of the 200 groundwater viral sequences with publicly available bacterial and archaeal viruses revealed that 85 groundwater viral genomes formed 41 viral clusters with at least one representative of groundwater virus (Table S3). Of these 41 clusters, 9 included a reference viral genome (Fig 2) and 32 were putative new viral genera. The details on the size of different clusters is depicted in Fig S2. The largest identified virus was a circular 296,356 bp contig (see virus size distribution depicted in Fig 3), and was part of a novel viral cluster. Although more viral sequences were identified from the background versus contaminated groundwaters, the fractions of all contigs

272 identified as viral sequence was similar across both sites (Fig S1). Thus, the 200 groundwater 273 viruses spanned a wide variety of sizes and included representatives of both known and novel viral 274 genera. 
276 Several aspects of the viral clusters provide evidence to optimal clustering of groundwater viruses.

277 All the 9 viral clusters with known reference viral genomes were circular DNA viruses. The VC

278 with 14 representatives had 11 representatives belonging to the family Microviridae, sub family

279 Gokushovirinae, which are 4.5-6kb, circular single stranded DNA virus. Interestingly, the 3 viral

280 contigs that are clustered are from the background site, and are also in the same size range (4.61,

2814.78 and $5.09 \mathrm{~kb}$ ). At least one virus (GW460_nc_scaffold_3616,8250bp size) from background

282 site is an inovirus $(5-15 \mathrm{~kb}$ size, circular single-stranded DNA genomes with rod-shaped or

283 filamentous virions ${ }^{46}$ ) clustering with known inovirus Ralstonia $\sim$ phage $\sim 1 \sim \mathrm{NP}-2014$. The genome

284 of inoviruses are known to be chromosomally integrated or replicated as a plasmid ${ }^{47}$, which may

285 be why this virus was recovered from plasmidome data.

286

287 Host Predictions

288 Once we identified viral genomes and their clusters, we sought to identify the range of hosts that these viruses infect. Using the 261 ORFRC bacterial isolates we were able to assign bacterial hosts to 20 viral genomes (Fig 4) out of the 200, indicating we were able to predict hosts for $10 \%$ of the

291 viral genomes identified (Table S4). As expected, the maximum number of predictions were made using tetranucleotide frequency (16), followed by BLAST (9) and CRISPR (2) analysis (Fig S3). All 9 viral sequences that had bacterial host genus predicted via BLAST, also had strain level predictions using BLAST99. An example of host prediction via BLAST99 is depicted in Fig S4 where the entire viral sequence was found in five different Acidovorax strains. Interestingly, 7 viral genomes were assigned hosts using both BLAST and tetranucleotide frequency methods and 6 of them were predicted to the exact same bacterial genus, increasing the confidence in their host prediction. Out of 20, 10 viral genomes had Pseudomonas predicted as its bacterial host, and overall 18 viral genomes were assigned to Proteobacteria. This could be attributed to the fact that out of 261 ORFRC isolates, over 50\% were Pseudomonads, and over 85\% were Proteobacteria, making it easier to identify them as host strains. Thus, several ORFRC bacterial genus and strains belonging to phyla Proteobacteria, Actinobacteria, and Firmicutes were predicted as hosts for the 303 viruses.

304

305 We also leveraged the complete archaeal and bacterial genome sequences available on NCBI, to 
307 archaeal strains. Using the 14,028 bacterial strains, host predictions could be made for about 36.5

$308 \%$ (73 out of 200) of the viral genomes, with a vast majority assigned to the phylum Proteobacteria

309 (Table S5). Other bacterial hosts were in the phyla Actinobacteria, Bacteroidetes, Firmicutes,

310 Chlamydiae and Chloroflexi. Again, the maximum number of predictions were made using

311 tetranucleotide frequency (71), followed by BLAST (5) analysis (Fig S3). The BLAST99 had no

312 hits, so strain specific bacterial host predictions were not made. Interestingly all 5 viral genomes

313 that had predictions with BLAST, also had predictions using tetranucleotide frequency method.

314 Although a higher number of viral sequences could be assigned to bacterial hosts using WGS from

315 NCBI compared to ORFRC, the probability of finding a host for every bacterial WGS tested was

316 higher with ORFRC strains (7.6\%) compared to NCBI strains $(0.5 \%)$, highlighting the benefits of

317 including bacterial strains from the same environment as the viral sequence itself. More

318 importantly, strain-specific host assignments could only be made using groundwater bacterial

319 isolates, and such high-resolution host assignment is important when designing experiments aimed

320 at isolating specific phages.

321

322 Together using the ORFRC and NCBI strains host predictions we were able to assign bacterial 323 hosts to $38 \%$ (76 out of 200) of the viral genomes (Fig 5). Around 17 viruses had host predictions

324 based on both ORFRC and NCBI strains (Fig S3), with the same bacterial phyla predicted as hosts

325 (Table S6). Differences like this could be attributed to the non-overlapping nature of the strains

326 from NCBI and ORFRC, and differences in the strength of host prediction methodologies. Next,

327 we compared host prediction between members of the same viral cluster (Table S7). The bacterial

328 host predicted mostly remained consistent within the same viral cluster. The minor discrepancy

329 seen in the viral clusters can likely be explained on further analysis, for instance the exceptional

330 viral cluster (VC_138_0), consists of ten members with six being groundwater viruses and their

331 hosts were predicted to be either Burkholderiales or Pseudomonadales based on the prediction

332 method used. Interestingly, the four known viruses they cluster with were Bordetella virus BPP1,

333 Pseudomonas phage AF, Pseudomonas phage vB_PaeP_Tr60_Ab31, and Xanthomonas citri

334 phage CP2 indicating members of this cluster infect both Burkholderiales and Pseudomonadales.

335 Thus, consistent patterns of host prediction emerge within the same viral cluster.

336

337 Presence of metabolic genes 
338 In addition to affecting groundwater biogeochemistry through their physical contribution to

339 dissolved organic matter and the lysis of their hosts, viruses can also affect the diversity and

340 function of microbial populations through the incorporation and expression of Auxiliary Metabolic

341 Genes $(\mathrm{AMGs})^{4,48}$. AMG definitions are still being refined ${ }^{49}$, but generally these genes are not

342 involved in viral replication or structure but instead allow viruses to directly manipulate host

343 metabolism during infection. Examination of all the viral sequences revealed a total of 1,486 hits

344 classified into known Pfam categories ${ }^{34}$ (Fig S5). Exploring Pfam domains associated with

345 microbial metabolism resulted in the identification of 51 unique putative AMGs (Table S8). Since

346 these viral sequences are from a site where metal and antibiotic resistance genes are routinely

347 seen ${ }^{17,50,51}$, all the unique PFAM hits were manually curated to identify metal and antibiotic

348 resistance genes. We found that the metal resistance genes identified as putative AMGs were those

349 providing resistance to copper, while the antibiotic resistance genes in the list of putative AMGs

350 were annotated as beta lactamase multi-resistance providing resistance to $\beta$-lactam antibiotics,

351 multi-drug efflux pumps AcrB/AcrD/AcrF family providing multi-drug resistance, and

352 streptomycin adenylyltransferase providing resistance to streptomycin. An excellent example is

353 viral sequence GW456_c_scaffold_130 which was annotated to encode metal and antibiotic

354 resistance genes along with signature phage genes consistent with a complete phage genome (Fig

355 6, annotation details in Table S9). The compilation of all the data discussed is available in Table

356 S10. To the best of our knowledge, this is the first report of the presence of metal and antibiotic

357 resistance genes on viral sequences. The presence of metal and antibiotic resistance genes suggests

358 that groundwater viruses may manipulate metal tolerance mechanisms enabling their hosts to adapt

359 to environmental stressors.

360

\section{Conclusion}

362 We demonstrate identification of novel viruses by leveraging plasmidome data for exploring

363 environmental viral communities. Our analyses revealed the presence of novel viruses, likely

364 representing new viral genera, in the underexplored groundwater environment. Using different

365 datasets, we achieved bacterial host predictions for a substantial number of the viral sequences.

366 Several of these phages encode genes related to signaling and tolerance mechanisms, thus likely

367 augmenting ecosystem function by modifying the metabolism of their bacterial hosts.

368 Interestingly, we find genes annotated to provide tolerance to metals, which is significant source 
369 of stress at this site. These predictions form the basis of future work on guiding phage isolation

370 efforts and functional assessment of virus-host linkages. The ability to isolate phages would open

371 new avenues for targeted manipulation of specific subsets of bacteria thus allowing for the

372 systematic dissection of a microbiome for probing community dynamics and function.

373

374

\section{Funding Sources}

375 This work was part of the ENIGMA- Ecosystems and Networks Integrated with Genes and 376 Molecular Assemblies (http://enigma.lbl.gov), a Science Focus Area Program at Lawrence 377 Berkeley National Laboratory and is supported by the U.S. Department of Energy, Office of 378 Science, Office of Biological \& Environmental Research under contract number DE-AC02379 05CH11231 between Lawrence Berkeley National Laboratory and the U. S. Department of 380 Energy. The work conducted by the U.S. Department of Energy Joint Genome Institute (SR) is 381 supported by the Office of Science of the U.S. Department of Energy under contract no. DE-AC02382 05CH11231. Part of the sequencing work by SS and EJA was funded by the National Cancer 383 Institute of the NIH under award P30-CA14051. The funders had no role in study design, data 384 collection and interpretation, or the decision to submit the work for publication. The United States

385 Government retains and the publisher, by accepting the article for publication, acknowledges that 386 the United States Government retains a non-exclusive, paid-up, irrevocable, world-wide license to 387 publish or reproduce the published form of this manuscript, or allow others to do so, for United 388 States Government purposes.

\section{Acknowledgements}

390 We thank Prof. Terry C. Hazen (University of Tennessee, Knoxville) for providing ORFRC 391 groundwater samples for the isolation of bacterial strains. We thank Austin Hendricks and Jon 392 Penterman for assistance as a part of the molecular biology MIT core facility; MIT 393 BioMicroCenter (https://openwetware.org/wiki/BioMicroCenter). We thank Albert Tang, 394 University of California, Berkeley for help with data analysis.

\section{Conflict of interest}

396 Authors do not have any conflict of interest. 


\section{References}

399 1. Sime-Ngando, T. Environmental bacteriophages: viruses of microbes in aquatic $400 \quad$ ecosystems. Front Microbiol 5, 355 (2014).

401 2. Brum, J.R. \& Sullivan, M.B. Rising to the challenge: accelerated pace of discovery 402 transforms marine virology. Nat Rev Microbiol 13, 147-159 (2015).

403 3. Rohwer, F. \& Thurber, R.V. Viruses manipulate the marine environment. Nature 459, 404 207-212 (2009).

405 4. Breitbart, M., Bonnain, C., Malki, K. \& Sawaya, N.A. Phage puppet masters of the 406 marine microbial realm. Nat Microbiol 3, 754-766 (2018).

407 5. Coutinho, F.H., Gregoracci, G.B., Walter, J.M., Thompson, C.C. \& Thompson, F.L. 408 Metagenomics Sheds Light on the Ecology of Marine Microbes and Their Viruses. 409 Trends Microbiol 26, 955-965 (2018).

410 6. Kauffman, K.M. et al. Viruses of the Nahant Collection, characterization of 251 marine $411 \quad$ Vibrionaceae viruses. Sci Data 5 (2018).

412 7. Andreani, J., Verneau, J., Raoult, D., Levasseur, A. \& La Scola, B. Deciphering viral 413 presences: two novel partial giant viruses detected in marine metagenome and in a mine 414 drainage metagenome. Virol J 15 (2018).

415 8. Yu, D.T., Han, L.L., Zhang, L.M. \& He, J.Z. Diversity and Distribution Characteristics of 416 Viruses in Soils of a Marine-Terrestrial Ecotone in East China. Microb Ecol 75, 375-386 (2018).

$4189 . \quad$ Weynberg, K.D. Viruses in Marine Ecosystems: From Open Waters to Coral Reefs. $A d v$ 419 Virus Res 101, 1-38 (2018).

420 10. Appelo CA, P.D. Geochemistry, groundwater and pollution. CRC press (2004).

421 11. Watson, D., Kostka, J., Fields, M. \& Jardine, P. The Oak Ridge field research center 422 conceptual model. NABIR Field Research Center, Oak Ridge, TN (2004).

423 12. Bruce, G.M., Flack, S. M., Mongan, T. R. \& Widner, T. E. Mercury releases from lithium 424 enrichment at the Oak Ridge Y-12 plant: A reconstruction of historical releases and off425 site doses and health risks. Reports of the Oak Ridge Dose Reconstruction (Tennessee 426 Department of Health) (1999). 
427 13. Rothschild, E.R., Turner, R.R., Stow, S.H., Bogle, M.A., Hyder, L.K., Sealand, O.M., \& 428 Wyrick, H.J. (1984).

429 14. Schulz, F. et al. Hidden diversity of soil giant viruses. Nat Commun 9 (2018).

430 15. Ahlgren, N.A., Fuchsman, C.A., Rocap, G. \& Fuhrman, J.A. Discovery of several novel, widespread, and ecologically distinct marine Thaumarchaeota viruses that encode amoC

433 16. Edwards, R.A. \& Rohwer, F. Viral metagenomics. Nat Rev Microbiol 3, 504-510 (2005).

434 17. Kothari, A. et al. Large Circular Plasmids from Groundwater Plasmidomes Span Multiple 435 Incompatibility Groups and Are Enriched in Multimetal Resistance Genes. MBio 10 436 (2019).

437 18. Roux, S., Enault, F., Hurwitz, B.L. \& Sullivan, M.B. VirSorter: mining viral signal from 438 microbial genomic data. Peerj 3 (2015).

439 19. Bolduc, B., Youens-Clark, K., Roux, S., Hurwitz, B.L. \& Sullivan, M.B. iVirus:

440 facilitating new insights in viral ecology with software and community data sets

441 imbedded in a cyberinfrastructure. Isme Journal 11, 7-14 (2017).

442 20. Bolduc, B. et al. vConTACT: an iVirus tool to classify double-stranded DNA viruses that $443 \quad$ infect Archaea and Bacteria. Peerj 5 (2017).

444 21. Shannon, P. et al. Cytoscape: A software environment for integrated models of 445 biomolecular interaction networks. Genome Res 13, 2498-2504 (2003).

446 22. Parks, D.H., Imelfort, M., Skennerton, C.T., Hugenholtz, P. \& Tyson, G.W. CheckM: 447 assessing the quality of microbial genomes recovered from isolates, single cells, and 448 metagenomes. Genome Res 25, 1043-1055 (2015).

449 23. Martin, M. Cutadapt removes adapter sequences from high-throughput sequencing reads. 450 EMBnet. journal 17, 10-12 (2011).

451 24. Bolger, A.M., Lohse, M. \& Usadel, B. Trimmomatic: a flexible trimmer for Illumina 452 sequence data. Bioinformatics 30, 2114-2120 (2014).

453 25. Bankevich, A. et al. SPAdes: A New Genome Assembly Algorithm and Its Applications 454 to Single-Cell Sequencing. J Comput Biol 19, 455-477 (2012).

455 26. Edgar, R. SINTAX: a simple non-Bayesian taxonomy classifier for 16S and ITS 456 sequences. BioRxiv, 074161 (2016). 
457 27. Lagesen, K. et al. RNAmmer: consistent and rapid annotation of ribosomal RNA genes.

458

459

460

461

462

463

464

465

466

467

468

469

470

471

472

473

474

475

476

477

478

479

480

481

482

483

484

485

486

487 Nucleic Acids Res 35, 3100-3108 (2007).

28. Cole, J.R. et al. Ribosomal Database Project: data and tools for high throughput rRNA analysis. Nucleic Acids Res 42, D633-D642 (2014).

29. Roux, S. et al. Minimum Information about an Uncultivated Virus Genome (MIUViG). Nat Biotechnol 37, 29-37 (2019).

30. Edwards, R.A., McNair, K., Faust, K., Raes, J. \& Dutilh, B.E. Computational approaches to predict bacteriophage-host relationships. Fems Microbiology Reviews 40, 258-272 (2016).

31. Andersson, A.F. \& Banfield, J.F. Virus population dynamics and acquired virus resistance in natural microbial communities. Science 320, 1047-1050 (2008).

32. Mizuno, C.M., Rodriguez-Valera, F., Kimes, N.E. \& Ghai, R. Expanding the Marine Virosphere Using Metagenomics. Plos Genet 9 (2013).

33. Roux, S., Hallam, S.J., Woyke, T. \& Sullivan, M.B. Viral dark matter and virus-host interactions resolved from publicly available microbial genomes. Elife 4 (2015).

34. Roux, S. et al. Ecogenomics and potential biogeochemical impacts of globally abundant ocean viruses. Nature 537, 689-+ (2016).

35. Bland, C. et al. CRISPR Recognition Tool (CRT): a tool for automatic detection of clustered regularly interspaced palindromic repeats. Bmc Bioinformatics 8 (2007).

36. Biswas, A., Gagnon, J.N., Brouns, S.J.J., Fineran, P.C. \& Brown, C.M. CRISPRTarget: Bioinformatic prediction and analysis of crRNA targets. Rna Biol 10, 817-827 (2013).

37. Ogilvie, L.A. et al. Genome signature-based dissection of human gut metagenomes to extract subliminal viral sequences. Nat Commun 4 (2013).

38. Marcais, G. \& Kingsford, C. A fast, lock-free approach for efficient parallel counting of occurrences of k-mers. Bioinformatics 27, $764-770$ (2011).

39. Edgar, R.C. MUSCLE: a multiple sequence alignment method with reduced time and space complexity. Bmc Bioinformatics 5, 1-19 (2004).

40. Kumar, S., Stecher, G. \& Tamura, K. MEGA7: Molecular Evolutionary Genetics Analysis Version 7.0 for Bigger Datasets. Mol Biol Evol 33, 1870-1874 (2016).

41. El-Gebali, S. et al. The Pfam protein families database in 2019. Nucleic Acids Res 47, D427-D432 (2019). 
488 42. Eddy, S.R. Accelerated Profile HMM Searches. Plos Comput Biol 7 (2011).

489 43. Gorlas, A., Krupovic, M., Forterre, P. \& Geslin, C. Living Side by Side with a Virus:

490 Characterization of Two Novel Plasmids from Thermococcus prieurii, a Host for the

491 Spindle-Shaped Virus TPV1. Appl Environ Microb 79, 3822-3828 (2013).

492 44. Arnold, H.P. et al. The genetic element pSSVx of the extremely thermophilic

493 crenarchaeon Sulfolobus is a hybrid between a plasmid and a virus. Mol Microbiol 34,

494 217-226 (1999).

495 45. Lima-Mendez, G., Van Helden, J., Toussaint, A. \& Leplae, R. Reticulate representation

496 of evolutionary and functional relationships between phage genomes. Mol Biol Evol 25,

$497 \quad 762-777$ (2008).

498 46. Ge, X.X. et al. Iron- and aluminium-induced depletion of molybdenum in acidic

499 environments impedes the nitrogen cycle. Environmental Microbiology 21, 152-163

500 (2019).

501 47. Waldor, M.K. \& Mekalanos, J.J. Lysogenic conversion by a filamentous phage encoding cholera toxin. Science 272, 1910-1914 (1996).

503 48. Breitbart, M., Thompson, L.R., Suttle, C.A. \& Sullivan, M.B. Exploring the Vast Diversity of Marine Viruses. Oceanography 20, 135-139 (2007).

505 49. Yooseph, S. et al. The Sorcerer II Global Ocean Sampling expedition: Expanding the $506 \quad$ universe of protein families. Plos Biol 5, 432-466 (2007).

507 50. Hemme, C.L. et al. Metagenomic insights into evolution of a heavy metal-contaminated 508 groundwater microbial community. Isme Journal 4, 660-672 (2010).

509 51. Hemme, C.L. et al. Lateral gene transfer in a heavy metal-contaminated-groundwater 510 microbial community. MBio 7, e02234-02215 (2016). 


\section{Figures with captions}

514

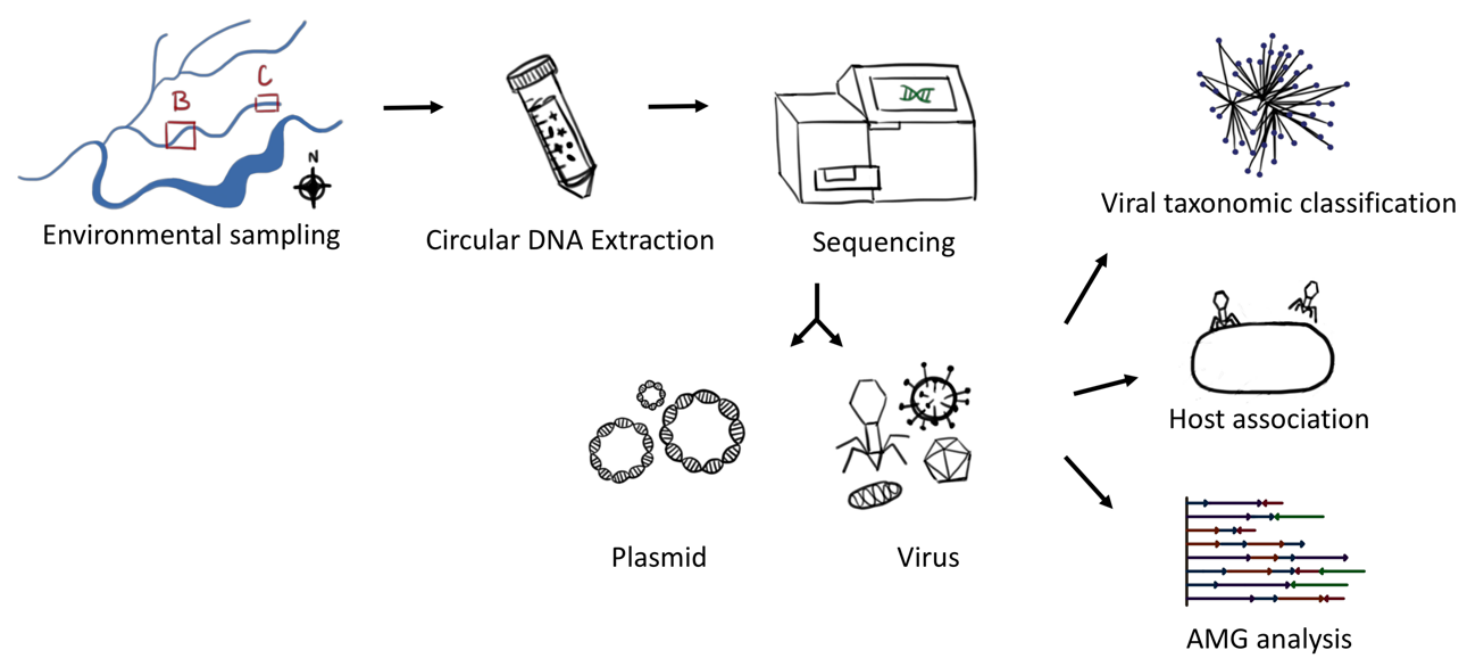

515

516 Fig 1: Overview of the study. Groundwater from the Oak Ridge Field research Site from

517 background (B) and contaminated (C) areas was filtered and subjected to circular DNA extraction.

518 Sequencing, assembly and annotation resulted in identification of both plasmids and viral

519 genomes. The viral genomes were subjected to viral cluster analysis to study the virus types, host

520 association analysis to get a prediction of bacteria they might infect and Auxiliary Metabolite

521 Analysis (AMG) analysis to study what functional genes they encode. 


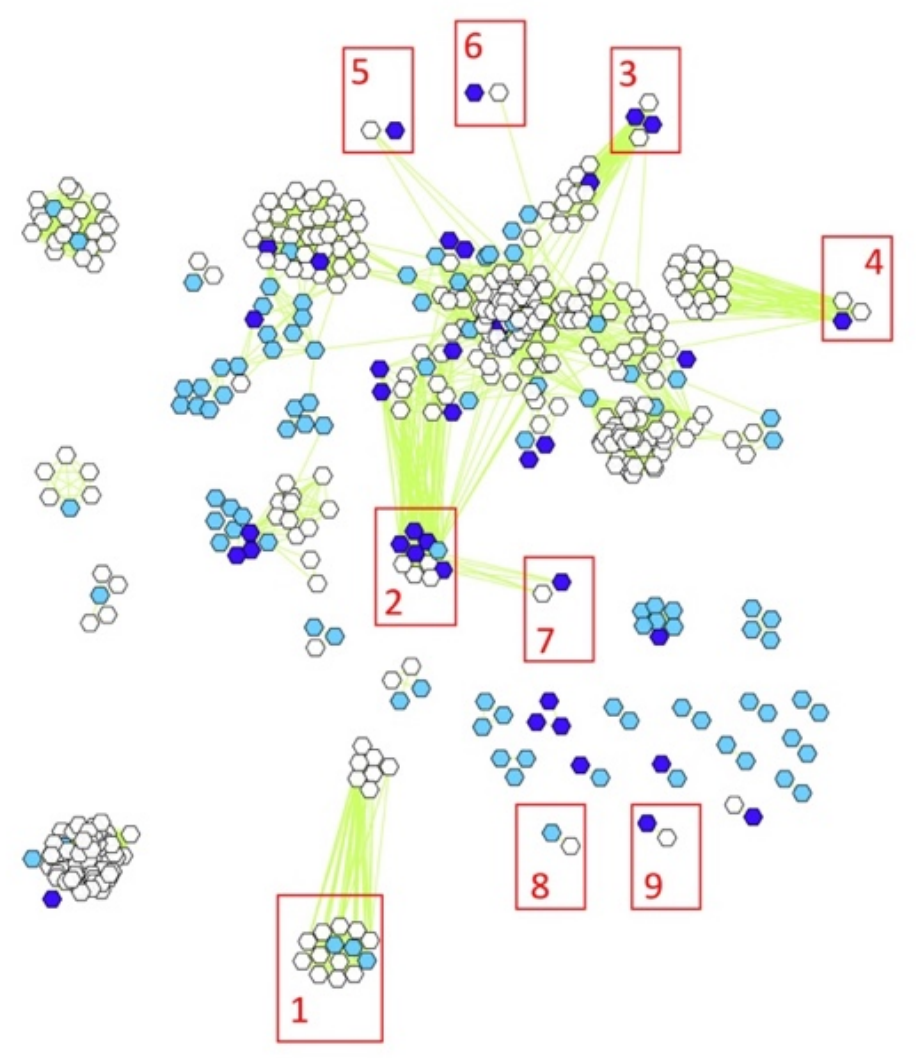

524 Fig 2: vContact generated viral cluster map depicting clustering of 85 viral sequences from 525 background (light blue) and contaminated (dark blue) groundwater, along with known virus 526 reference genomes (white). The 9 viral clusters that contain known viruses are annotated on the 527 figure as 1) Microviridae 2) Podoviridae (Caudovirales) 3) Myoviridae (Caudovirales) 4) 528 Myoviridae (Caudovirales) 5) Podoviridae (Caudovirales) 6) Siphoviridae (Caudovirales) 7) 529 Podoviridae (Caudovirales) 8) Inoviridae and 9) Myoviridae (Caudovirales). The order and 530 distance between different viruses is an arbitrarily selected value. 
bioRxiv preprint doi: https://doi.org/10.1101/2020.07.14.203604; this version posted July 15, 2020. The copyright holder for this preprint (which was not certified by peer review) is the author/funder, who has granted bioRxiv a license to display the preprint in perpetuity. It is made available under aCC-BY-NC-ND 4.0 International license.

531

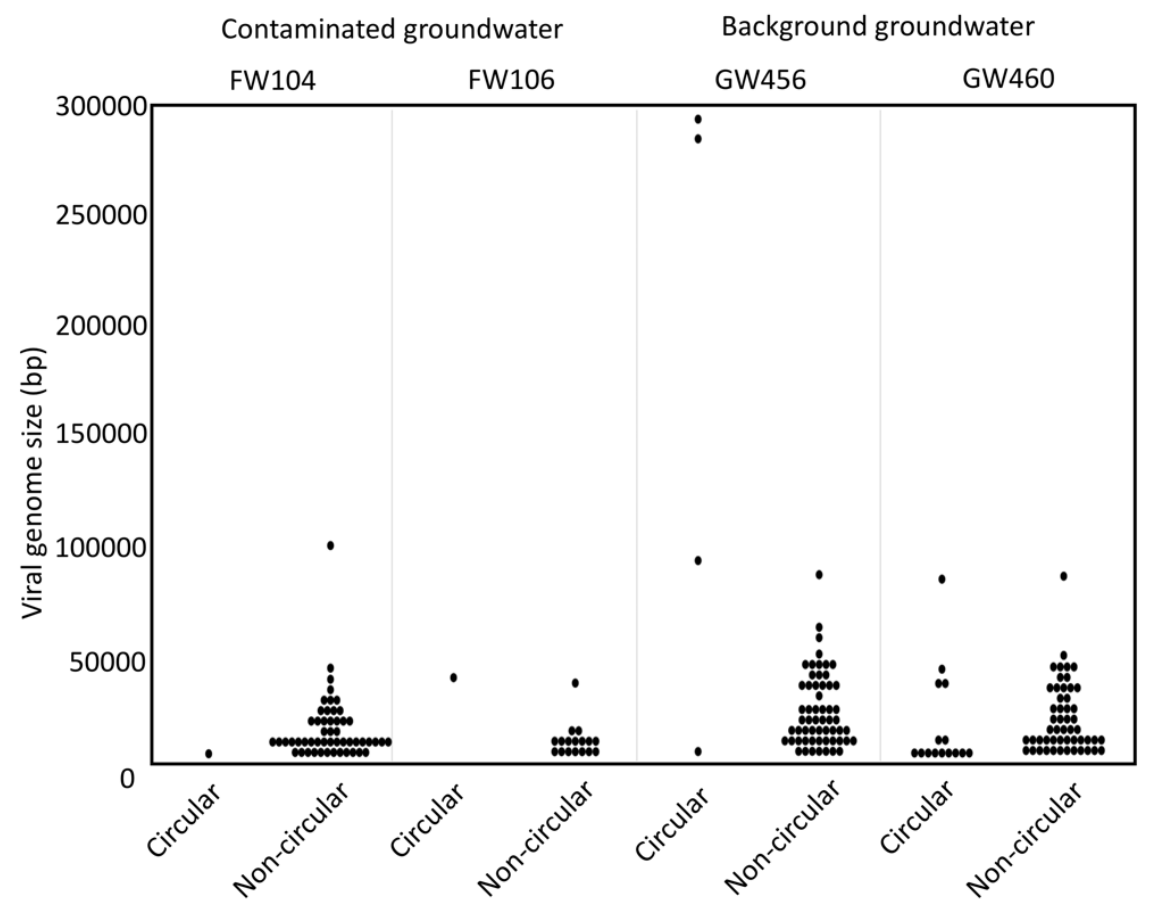

532

533 Fig 3: Size distribution of viruses from the background and contaminated groundwaters.

534
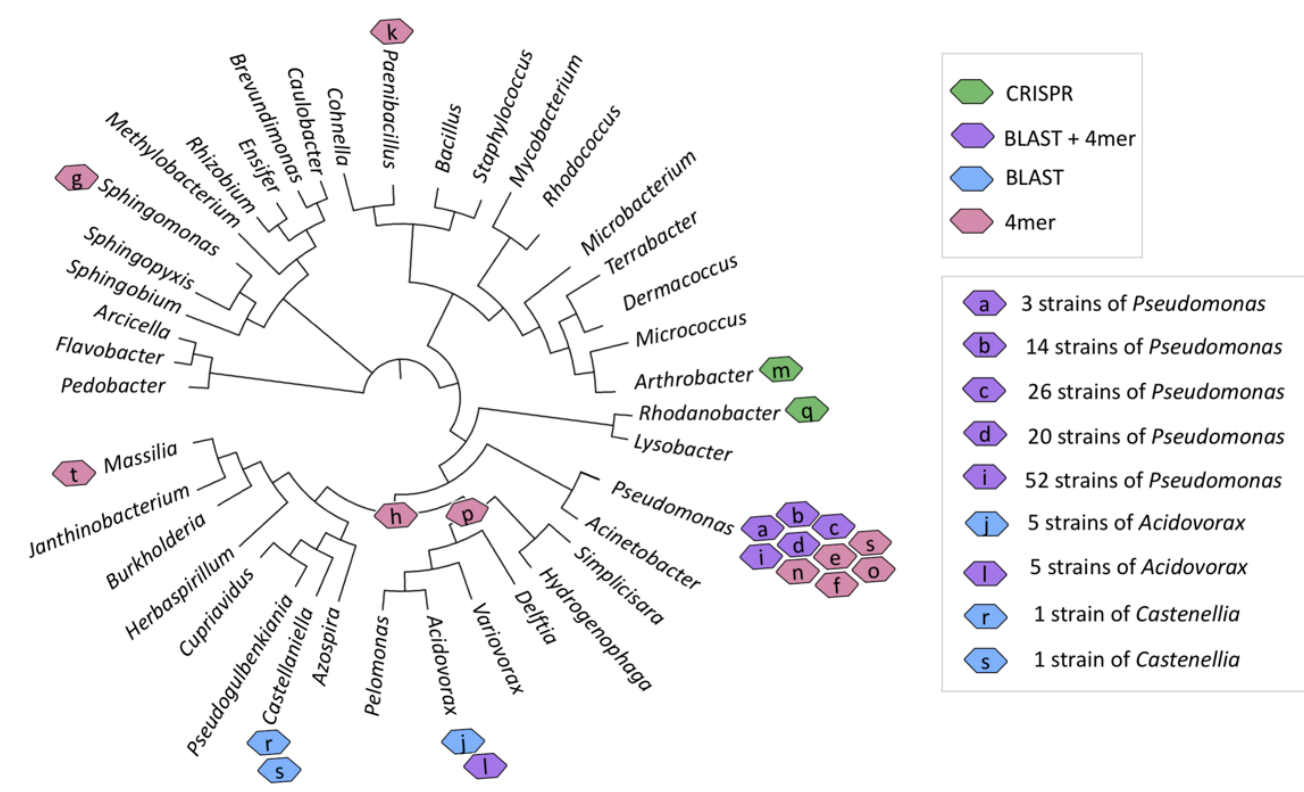

535 
537 Fig 4: Viral host predictions based on BLAST, high stringency BLAST (BLAST99), 538 tetranucleotide frequency (4mer) and CRISPR methods using whole genome sequence(WGS) 539 information from 261 ORFRC bacterial isolates. The details of the 20 viruses ("a"-“t”) are 540 provided in Table S4. The viruses " $\mathrm{h}$ ", and " $\mathrm{p}$ " have their hosts assigned to Class

541 Betaproteobacteria and Family Comamonadaceae. The rest of the viruses are assigned to the 542 genera. The phylogenetic tree was made from 16S rRNA sequence of 261 ORFRC isolate strains.

543 The viral sequence "s" appears twice because it was predicted to infect two different genera based 544 on the different prediction methods.

545

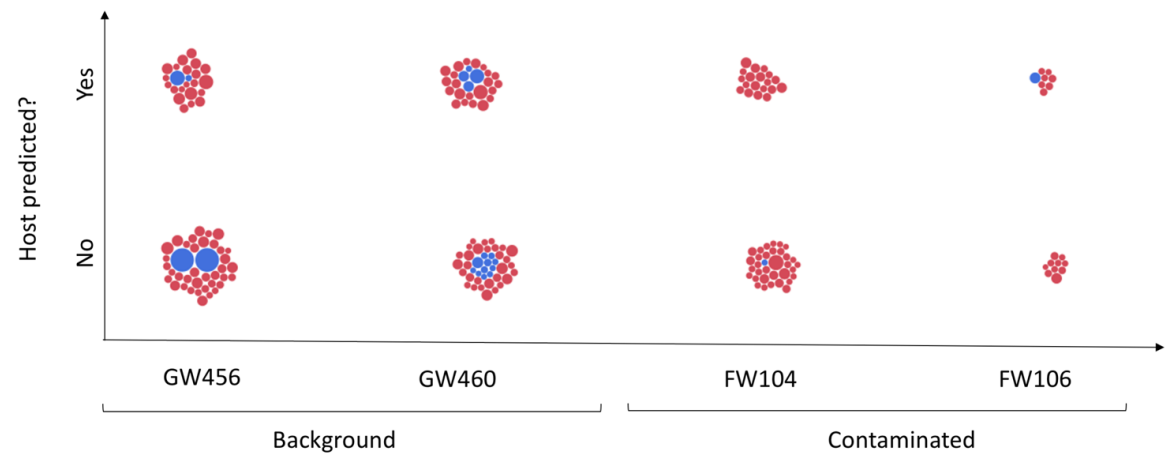

546

547 Fig 5: Compilation of viral sequences from the groundwater sites based on availability of bacterial 548 host prediction. Circular viral sequences are depicted in blue, while the rest are in red. The size of 549 the circle is indicative of the viral sequence size. 


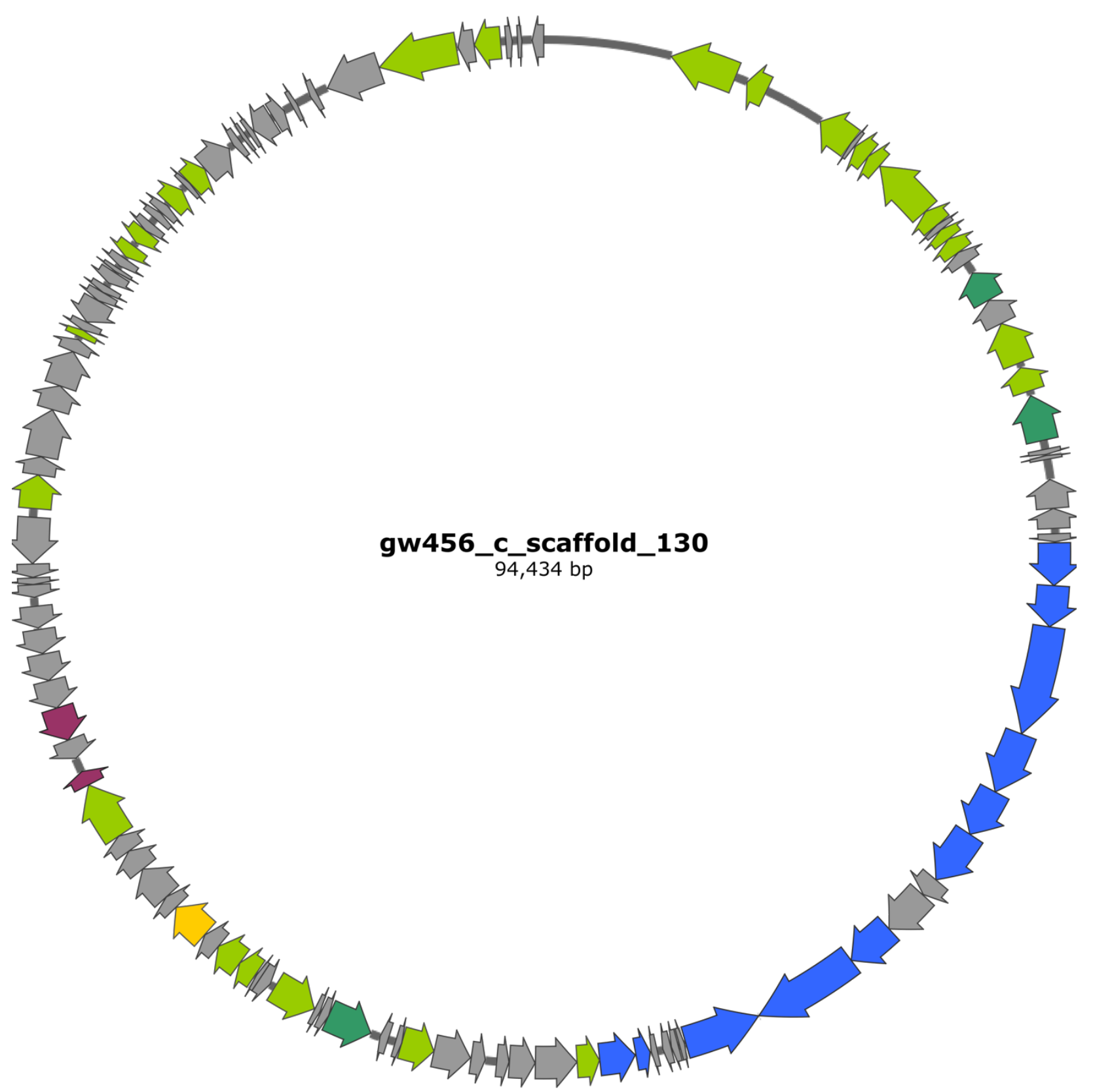

552 Fig 6: Example of a viral contig carrying auxiliary metabolite genes. Map of the virus 553 (gw456_c_scaffold_130) from background groundwater with phage-related genes highlighted in 554 green (darker green represents true hallmark genes of viruses), metal (copper, cobalt, zinc, 555 cadmium, lead, mercury, arsenic) resistance genes highlighted blue, antibiotic (spectinomycin and 556 fosfomycin) resistance genes highlighted in pink and metabolism (lactate dehydrogenase) gene in 557 yellow. The viral contig was annotated via Prokka in Kbase $^{54}$ and the annotation for virus558 associated genes were updated on the map using virSorter ${ }^{18}$ predictions, details in Table S10. 


\section{Supplementary Information Figures}

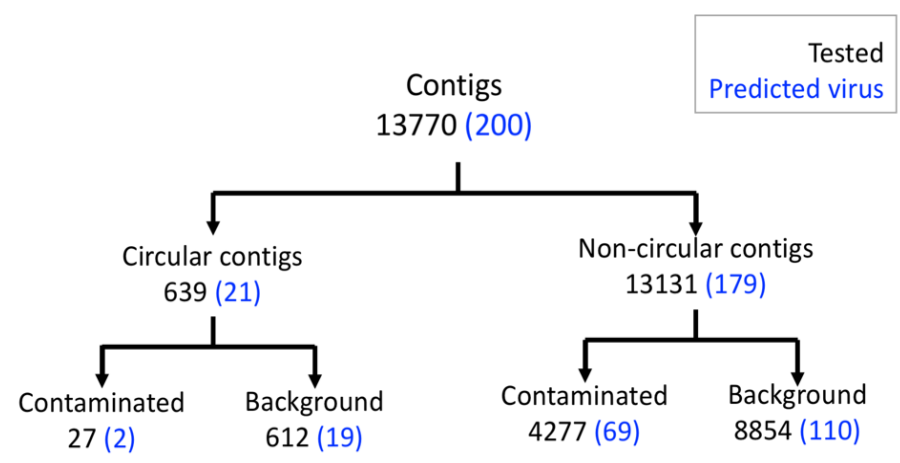

561

562

563

564 Fig S1: Breakdown of the all the contigs tested, with the those predicted to be viral highlighted in

565 blue.

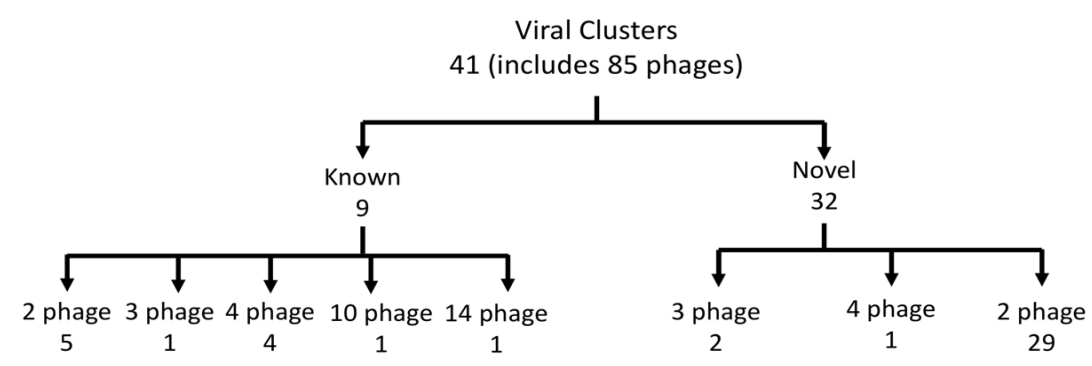


568 Fig S2: Distribution of viral clusters and the number of phages in each cluster.

569

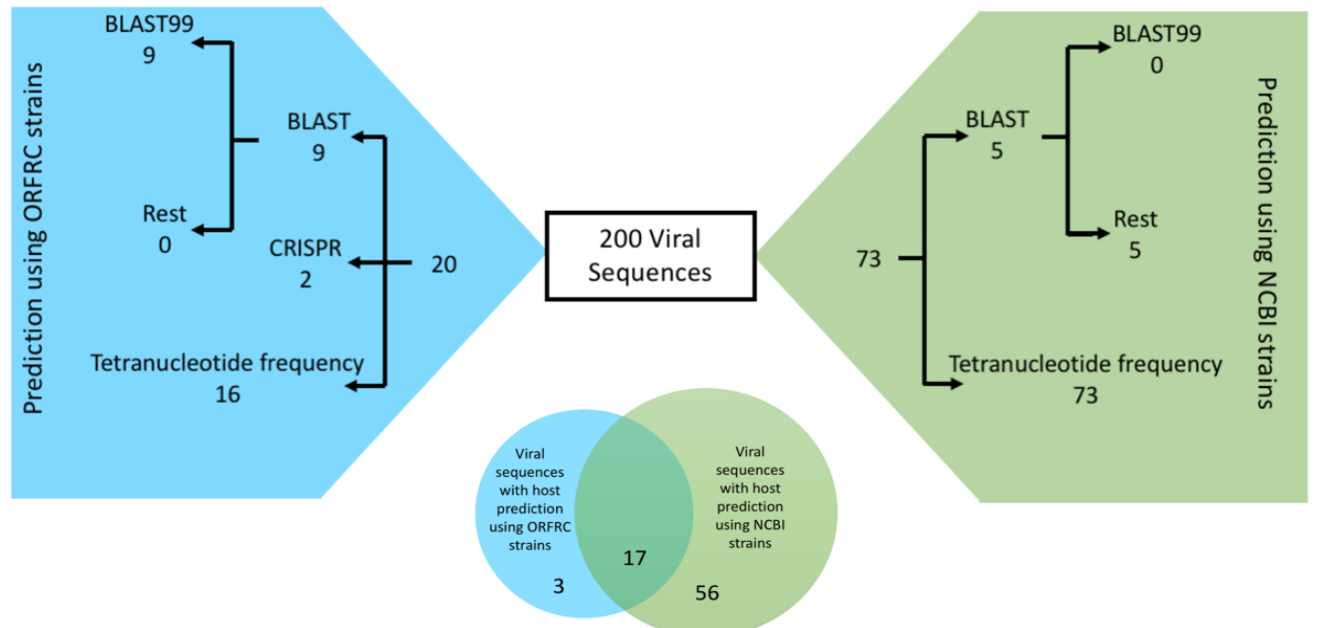

570

571

572 Fig S3: Details of numbers of viral sequences for which hosts are predicted using various bacterial

573 host prediction methods on ORFRC and NCBI strains with whole genome sequences. Venn

574 diagram shows the overlap of host prediction for viral sequences when using the ORFRC and 575 NCBI strains. 
578 Fig S4: Results of host association for virus GW456_nc_scaffold_4557. About 99.9\% of the viral sequence $(\mathrm{e}-\mathrm{value}=0)$ could be found in 5 Acidovorax strains isolated from ORFRC.

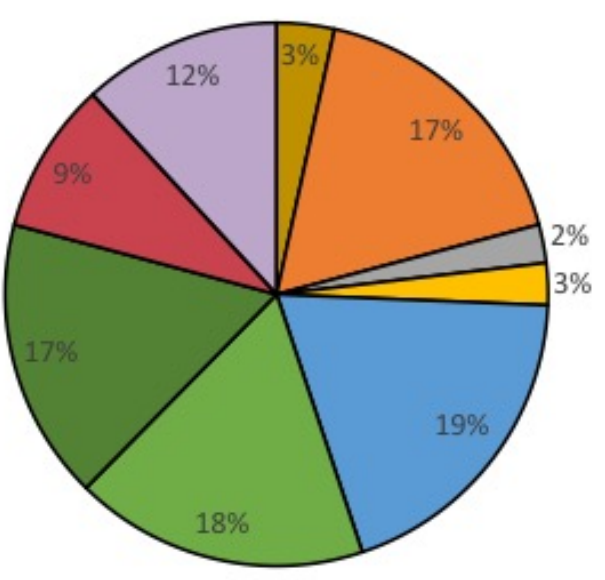

口AMG (metabolism)

口DNA replication, recombination, repair; nucleotide metabolism

口lysis

$\square$ membrane transport

口Not categorized

口other

口structural

口transcription, translation, protein synthesis

口unknown

582 Fig S5: Major categories of 1486 virus encoded genes predicted via Pfam database. The hits were sorted into categories based on gene names as previously established based on AMGs seen in ocean viruses ${ }^{34}$.

Table S1: This file contains information on all contigs identified as viruses. These are sorted into 5 and considered as phage in this study.

Table S2: This file contains details of the 261 bacterial strains isolated from ORFRC.

Table S3: This file contains information on all 41 viral clusters that groundwater viruses fall into.

596 This includes 32 novel clusters and 9 known clusters including known phages.

598 Table S4: Host association predictions based on ORFRC groundwater bacterial whole genome sequences. 
601 Table S5: Host association predictions based on NCBI bacterial whole genome sequences.

602

603 Table S6: Breakdown of host assignments made using NCBI and ORFRC bacterial whole genome 604 sequences.

605

606 Table S7: Comparison of host prediction between members of the same VC using NCBI and

607 ORFRC bacterial whole genome sequences.

608

609 Table S8: Details of all the Pfam domains detected on the 200 viral sequences. Pfam domains

610 related to metal resistance, antibiotic resistance and toxin-antitoxin encoding genes are also listed.

611

612 Table S9: Details of the 121 genes encoded on the viral sequence GW456_c_scaffold_130

613 including their location, size, directionality and description.

614

615 Table S10: Details of all 200 viral sequences with compilation of all analyses including virus size,

616 viral cluster, host prediction, and AMG analysis.

617 